\title{
WEIGHTS FOR PREDICTION OF EARTH ORIENTATION PARAMETERS
}

\author{
N. Sekiguchi \\ Tokyo Astronomical Observatory \\ Mitaka, Tokyo 181 \\ Japan
}

\begin{abstract}
The most important factor in getting accurate predictions of Earth orientation parameters (EOP) is choosing suitable input data. Also, we must choose a proper statistical model for the noise in these data. The weights of the data are determined on the basis of these models. Finally, we must determine suitable values of parameters in the computation of EOP predictions.
\end{abstract}

\section{INTRODUCTION}

At the Tokyo Astronomical Observatory, we have published bi-monthly the predicted values of UT1-UTC for the coming month in the Preliminary Report, Series 1, Part II of the Time Division. I will discuss the process of routine prediction of UT1-UTC, $x$ and $y$.

\section{SUITABLE INPUT DATA FOR PREDICTIONS}

The EOP are a set of three random variables. There are remarkable differences in the statistical natures of UT1-UTC, and the polar coordinates $x$ and $y$. Therefore, it is impossible to treat those two cases in a similar manner. But in both cases, it is important to choose an adequate set of input values in order to get the best prediction results. The most suitable data for prediction are not necessarily the most accurate data. If we use inappropriate input data, it becomes very difficult to obtain accurate predictions, no matter how we improve the method of extrapolation. The best input for the predictions is data which has been passed through suitable filters so that it is free from certain spectral components. The spectral region of interest depends on the length of time between the last observation and the epoch of prediction.

The best input data can be chosen empirically. These will be the data which result in the residuals of the predicted values from the physical realization being minimized. For both UT1-UTC and pole positions, it is desirable to use the most recent possible observations. Our experience suggests that the Daily Interpolated Values of UT1-UTC from the BIH Rapid Service is the best input for extrapolation of UT1-UTC. However, the Rapid Service $\mathrm{x}$ and $\mathrm{y}$ values are not suitable for prediction of pole positions. For this purpose, the most useful data are the smoothed (final) values of $x$ and $y$ for every 5 days from BIH Circular D. (The combination of different kinds of input data always yielded inferior results.) 


\section{THE ANNUAL TERMS}

Needless to say, the annual terms, which are sufficiently stable, should be extracted from the observational data before the extrapolation. It is not a good idea, however, to use the UT2-UTC values obtained simply by extracting the officially adopted values for the annual component from UT1-UTC data. The values of the annual component should be determined every year from the observations of several recent years.

\section{EXTRAPOLATION OF UT1-UTC}

The most suitable extrapolation procedures are different for the cases of time and polar motion. For the prediction of UT1-UTC, linear extrapolation gives the best results in our experience. Parabolic, or algebraic extrapolation, and extrapolation by trigonometric functions both produced inferior results.

The noise model for UT1-UTC is in close conformity with the ones proposed by Brouwer ${ }^{[1]}$ and Van Woerkom ${ }^{[2]}$ for very long time intervals ${ }^{[3]}$. According to them, the rate of UT reveals itself as an accumulated random walk, in which the steps are mutually independent. They proposed, therefore, that the weights for fitting a straight line to these data should be proportional to the cubic inverse of the time intervals between observations. But the actual observations contain observational errors. Since the weight should be proportional to the reciprocal of the dispersion value, we can adopt an expression for the weight of the form

$$
W=1 /\left[1+p\left(t_{0}-t\right)^{3}\right]
$$

where $p$ is a parameter, $t_{0}$ is the epoch of the last data, and $t$ is the epoch of the individual data. This expression for the weight gives the best results for the extrapolation of UT1UTC. The best value of the parameter $p$ is determined empirically.

\section{EXTRAPOLATION OF POLE POSITIONS}

For the prediction of pole positions, $\mathrm{x}$ and $\mathrm{y}$, our experiences indicate that extrapolation of the circular motion gives the best results. In this case, we adopted the model of random walk for excitation of polar motion. We assumed that the pole position $\underline{m}=x+i y$ (the underline indicates a complex number) can be expressed as

$$
\underline{m}-\underline{\phi}_{0}=\left(\underline{m}_{0}-\underline{\phi}_{0}\right) \exp \left(i c\left(t-t_{0}\right)\right)
$$

where $i$ is the imaginary unit, $c$ is the Chandlerian frequency, $\phi$ is the position of the excitation pole, and $\underline{m}_{0}$ and $\underline{\phi}_{0}$ are the positions of the pole and the excitation pole at the epoch respectively. When we assume that the motion of the excitation pole has the nature of random walk, we can show that the reciprocal of the dispersion of the deviation from the standard motion (2) has the form of (1) approximately ${ }^{[4]}$. Therefore, we can assume that the best weights for prediction of UT1-UTC and pole positions are of form (1) in both cases.

\section{VALUES OF PARAMETERS}

For the values of the parameter $p$, we adopted $p=0.13 /(\text { day })^{3}$ for the prediction of UT1UTC, and $p=0.020 /(\text { day })^{3}$ for pole predictions. These values should be redetermined from 
time to time because the quality of the data will vary over the years. But improvements in the values of the parameter $p$ have only a slight effect on the accuracy of the predictions, in our experience.

As for the length of the input data set, the longer the better. Normally, we use observations from the past year for the prediction of UT1-UTC and pole position. We have not published the latter predictions.

\section{ACCURACIES}

The standard deviations of the predicted values from the definitive values of the BIH Circular D are as follows. For UT1-UTC, the standard deviation is 3 milliseconds with an elapsed time of 12 days from the last date of input data, and 12 milliseconds with an elapsed time of 38 days. For pole positions, the standard deviation is $0^{\prime \prime} .013$ with an elapsed time of 75 days.

\section{REFERENCES}

[1] Brouwer, D. 1952, 'A study of change in the rate of rotation of the Earth', Astron. J. 57, 125-146.

[2] Van Woerkom, A. J. J., 1953, 'On cumulative sums of random numbers', Astron. J. 58, 10-20.

[3] Sekiguchi, N., 1984, 'Cumulative rate changes in the rotation of the Earth', J. Geodetic Society of Japan 30, 87-91.

[4] Sekiguchi, N. 1985, 'A Method of the prediction of the polar motion', J. Geodetic Society of Japan 31, 236-240.

\section{DISCUSSION}

Ye Shu-hua: The Shanghai Observatory has had a routine UT1 prediction for more than 4 years. The predictions are based on 10 optical instruments, using data 12 months before the date, and using AR modelling. The rms of UT1 predictions 10 days ahead, updated weekly, is $\pm 2 \mathrm{~ms}$. 